\title{
Design Practices in Harmonic Analysis Studies Applied to Industrial Electrical Power Systems
}

\author{
S. F. Mekhamer \\ Faculty of Engineering, \\ Ain Shams University \\ Cairo, Egypt \\ saidfouadmekhamer@yahoo.com
}

\author{
A. Y. Abdelaziz \\ Faculty of Engineering, \\ Ain Shams University \\ Cairo, Egypt \\ almoatazabdelaziz@hotmail.com
}

\author{
S. M. Ismael \\ Electrical Engineering Division, \\ ENPPI \\ Cairo, Egypt \\ shriefmohsen@enppi.com
}

\begin{abstract}
Power system harmonics may cause several problems, such as malfunctions of electrical equipment, premature equipment failures and plant shutdowns. Accordingly, mitigation of these harmonics is considered an important target especially for industrial applications where any short downtime period may lead to great economic losses. Harmonic analysis studies are necessary to analyze the current and voltage harmonic levels and check if these levels comply with the contractual or international standard limits. If the studies reveal that the preset limits are exceeded, then a suitable harmonic mitigation technique should be installed. Harmonic analysis studies in the industrial electrical systems are discussed in many references. However, a comprehensive procedure for the steps required to perform a harmonic study is rarely found in the literature even though it is strongly needed for design engineers. This paper provides a comprehensive procedure for the steps required to perform a harmonic study in the form of a flowchart, based on industrial research and experience. Hence, this paper may be considered as a helpful guide for design engineers and consultants of the industrial sector.
\end{abstract}

Keywords-harmonic analysis study; distortion; point of common coupling (PCC); variable frequency drive (VFD); resonance

\section{INTRODUCTION}

Due to the dramatic increase in the usage of nonlinear loads in industrial applications (mainly regarding Variable Frequency Drives or VFDs), the power system harmonics problems has gain in significance, representing a big obstacle against the wide application of VFDs although they enhance system efficiency and provide great energy saving. The power system harmonics cause many harmful effects including:

- Overheating of generators, motors, transformers, and power cables that lead to early equipment failures

- Failure of capacitor banks

- Nuisance tripping to protection relays

- Interference to communication systems and sensitive electronic devices

Accordingly, the mitigation of the power system harmonics is of great importance in industrial electrical systems in order to increase system reliability, enhance operation economics, avoid unwanted equipment failure and process downtimes [1]. Nowadays, industrial electrical systems contain a valuable amount of nonlinear loads. Accordingly, power system studies for industrial plants should contain harmonic analysis studies beside short circuit, load flow and motor starting studies. The harmonic analysis studies for the industrial systems are discussed in [2], but the author did not focus on the guidelines of the harmonic study. Also the authors did not introduce the various international standards that set the limits of the harmonic distortions.

The goals of this paper can be summarized as follow:

1. To highlight the purpose of a harmonic analysis study

2. To highlight some guidelines for harmonic analysis studies

3. To provide a comprehensive description of the procedure required to perform a harmonic study

4. To introduce the international standards limits for the harmonic distortions

\section{PURPOSE OF A HARMONIC ANALYSIS STUDY}

Nowadays, the applications of the nonlinear loads in the industrial plants grow rapidly and the percentage of these loads may be in the range of $30 \%$ to $50 \%$ of the total plant load. Accordingly, the effects of harmonics within the electrical system and their impact on the electric utility and neighboring plants should be examined to avoid equipment damage and plant shutdowns. The following cases may necessitate performing a harmonic study [3]:

1. During the design stage of a project, if the amount of the nonlinear loads exceeds $25 \%$ of the total loads on a bus or a system, a harmonic analysis study is required to check the compliance with the contractual/ international harmonic limits

2. To solve harmonic-related problems such as failure of electrical equipment or malfunction of protective relays

3. If an existing plant is going to be expanded and a significant amount of nonlinear loads is going to be added, then a harmonic analysis study is required to verify the plant performance after the addition of these loads 
4. If a capacitor bank is installed in any electrical networks that contain many nonlinear loads, then a harmonic analysis is required to check the possibility of resonance occurrence

\section{GUIDELINES FOR HARMONIC ANALYSIS STUDIES}

\section{A. Harmonic Sources}

All nonlinear loads are defined also as harmonic sources, as clearly shown in Figure 1, because they draw non-sinusoidal currents when a sinusoidal voltage is applied. The nonlinear load acts as a source of harmonic currents in power system, thus causing voltage distortions at the various system buses due to the harmonic voltage drops across the system impedances.

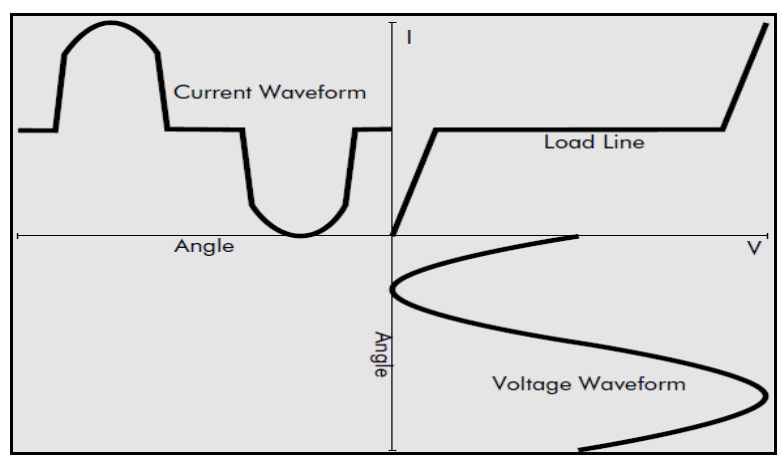

Fig. 1. Effect of a nonlinear load on the current waveform

To perform a harmonic study, the design engineer must identify the available harmonic sources and the harmonic currents generated by these harmonic sources. There are three options available for the design engineer to determine the harmonic currents, as described below:

a. To measure the generated harmonics from each harmonic source (time-consuming option, applicable only in case of existing plants)

b. To calculate the generated harmonic currents by using suitable mathematical analysis (may require extensive manual and time-consuming calculations)

c. To use typical values based on computerized softwares libraries or based on the available data from the nonlinear load's manufacturer

Practically, options (a) and (c) are the most used options and provide reasonable results.

The following are the main sources of harmonics in industrial applications [4]:

1) Saturable Magnetic equipment:

There are various saturable magnetic equipment that cause harmonic problems such as:

a. Rotating machines, rotating machines like induction motors may act as sources of the third harmonic currents when they are operating in abnormal or overloaded conditions. b. Ballasts of discharge lamps, the discharge lamps like mercury vapor, high-pressure sodium and fluorescent lamps are dominant sources of the third harmonic currents.

c. Transformer harmonics, transformers create harmonics when they are overexcited. In addition, the transformer inrush currents may contain some even harmonics, but the duration is rather limited.

d. Generator harmonics, voltage harmonics are created from the synchronous generators due to the non-sinusoidal distribution of the flux in the air gap. Selection of suitable coil-span factor (called also pitch factor) can significantly reduce the voltage harmonics from the generators.

2) Power Electronic Devices:

There are various power electronic devices that cause harmonic problems such as:

a. Variable Frequency Drives (VFDs) used in fans and pumps

b. Switched mode power supplies (SMPS), used in instruments and personal computers

c. High voltage DC transmission stations (HVDC)

d. Static VAR compensators

e. Uninterruptable power supply systems (UPS)

f. Battery charger systems

g. Flexible AC transmission systems (FACTS)

h. AC and DC arc furnaces in steel manufacturing plants

\section{B. Resonance}

The inductive reactance increases as the frequency increases as follow:

$$
X_{L}=2 . \pi \cdot F . L
$$

where:

$X_{L}$ : Inductive reactance

$F$ : System frequency

$L:$ Inductance

While the capacitive reactance decreases as the frequency increases as follows:

$$
X_{C}=1 /(2 . \pi . F . C)
$$

where:

$X_{C}$ : Capacitive reactance

$C$ : Capacitance

Due to the opposite characteristics of the inductive and capacitive reactances, there must be a frequency at which $X_{L}$ equals $X_{C}$. This condition of equal and opposite reactances is called "resonance". Most of the power system elements are inductive. Accordingly, the presence of shunt capacitors used 
for power factor correction or harmonic filtering can increase the probability of resonance occurrence. There are two types of resonance, the series resonance and the parallel resonance. The harmful effect of the series resonance may be the flow of excessive harmonic currents through the network elements. These excessive currents cause nuisance tripping to the protection relays, overheating of cables, motors, and transformers and premature failure to the electrical equipment. The harmful effect of the parallel resonance may be the presence of excessive harmonic voltages across the network elements. These excessive harmonic voltages cause dielectric breakdown of the electrical equipment's insulation [3].

\section{1) Series Resonance:}

The series resonance occurs when an inductor and a capacitor are connected in series and they resonate together at a certain resonance frequency. An example of the series resonant circuit is shown in Fig. 2. This AC circuit is said to be in resonance when the inductive reactance $X_{L}$ is equal to the capacitive reactance $X_{C}$.

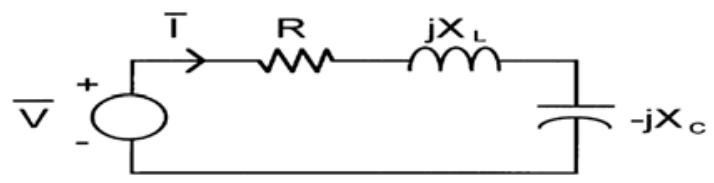

Fig. 2. AC circuit representing an example for the series resonance

\section{2) Parallel Resonance:}

The parallel resonance occurs when an inductor and a capacitor are connected in parallel and they resonate together at a certain resonance frequency. There are many forms of parallel resonant circuits. A typical parallel resonant circuit is shown in Figure 3. This circuit is said to be in parallel resonance when $X_{L}=X_{C}$ similar to the series resonance.

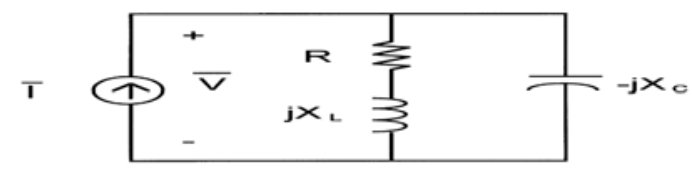

Fig. 3. AC circuit representing an example for the parallel resonance

C. Tools of performing a harmonic analysis study:

The harmonic analysis study can be performed by any of the following tools:

a. Manual calculations, which are limited to small-size networks since they are very complicated and susceptible to errors.

b. Field measurements, which are often used as a verification of the design, or as a preliminary diagnosis of a field problem.

c. Digital computer simulations, which nowadays are the most convenient and economical method for analyzing system harmonics

\section{Power System Modeling:}

At the presence of harmonics, the electrical system elements models must be updated to encounter for the presence of higher frequencies in the system rather than the power frequency $(50 \mathrm{~Hz}$ or $60 \mathrm{~Hz})$. Details for electrical system elements models under harmonics distortions can be found in [3].

\section{E. Types of Analyses Performed during the Harmonic} Analysis:

There are two main types of analyses that could be performed during harmonic analysis [2]:

a. Current and voltage distortion analysis, in which the individual and total current and voltage harmonic distortions are calculated at the various buses then the results are compared with the relevant contractual limits.

b. Impedance versus frequency analysis, in which a plot of the system impedance at various buses is plotted against the frequency. This analysis is important in predicting the system resonances prior to energizing the electrical system. A peak in the impedance plot indicates a parallel resonance while a valley in the impedance plot indicates a series resonance.

\section{STEPS OF PERFORMING A HARMONIC ANALYSIS STUDY}

If a harmonic analysis study is required to be performed due to any of the cases described in section (II), the following steps should be followed:

a. Obtain the electrical system one-line diagram and highlight the available nonlinear loads, capacitor banks and medium voltage cables of long length within the industrial system.

b. Highlight the point of common coupling (PCC) which is the point that connects the industrial network with the utility or with the neighboring plant.

c. Highlight the in-plant system buses that are expected to be affected from harmonic distortions.

d. Gather the harmonics-related data of all nonlinear loads within the plant.

e. Obtain, from the utility company, the relevant data of current and voltage harmonics at the contractual PCC including the minimum and maximum short circuit fault levels and the permissible limits on voltage and current harmonics because the allowable harmonic limits vary from country to country.

f. Model the electrical network using any of the commercially available softwares such as the electrical transient analyzer program (ETAP).

g. Perform the harmonic analysis for the electrical network at the various possible operating scenarios.

h. Check the individual and total voltage and current distortion levels at the interested system buses and at the PCC. 
i. Check the harmonic frequency spectrum, which is a plot of each individual harmonic value with respect to the fundamental value versus frequency.

j. If the harmonic distortion results exceed the allowable limits, select an appropriate harmonic mitigation solution and the optimum insertion point for that solution. Further details about this point are introduced in section (V).

k. Re-perform the harmonic analysis study after adding the harmonic mitigation technique to ensure compliance with the contractual / international harmonic limits.

An extensive literature review over the past twenty years leads to the fact that there is no single article that summarizes the steps required to perform a harmonic analysis study even though the importance of this procedure for the design engineers. The comprehensive flowchart presented in Figure 4 provides this novel helpful approach.

\section{SELECTION OF THE HARMONIC FILTER'S INSERTION POINT}

Even if the design engineer selects the optimum harmonic mitigation technique for his plant, among the available harmonic mitigation solutions in the market [5], then the filter insertion point should be studied carefully as it greatly affects system performance [6]. As shown in Figure 5, the possible filter insertion points can be classified into three categories as follow:

\section{A. Local Harmonic Mitigation}

In this mode of mitigation, the shunt type (passive or active) harmonic filter is directly connected to the nonlinear load terminals. This mode is efficient if the number of nonlinear loads is limited and the power of each nonlinear load is significant compared to the total plant power. Circulation of harmonic currents in the electrical network is avoided, thus the harmonic impact on the upstream network elements is minimized.

\section{B. Semi-Global Harmonic Mitigation}

In this mode of mitigation, the shunt type (passive or active) harmonic filter is connected to the input of the LV subdistribution switchboard. Accordingly, the filter treats several sets of nonlinear loads. This type of compensation is ideal in presence of multiple nonlinear loads each having low rated power. A practical example of this mode is found in commercial buildings where a harmonic filter may be found on each floor of the building.

\section{Global Harmonic Mitigation}

This mode of mitigation is more concerned with meeting the contractual harmonic limits at the (PCC) than the reduction of the in-plant harmonics. The major drawback of this mitigation mode is that the harmonic currents are allowed to circulate in the electrical network. Thus, the various electrical elements within the plant will be subjected to harmful harmonic impact.

\section{INTERNATIONAL HARMONIC STANDARDS}

The purpose of imposing strict limits on the harmonics emissions is to ensure that the current and voltage distortions at the PCC are kept sufficiently low. Thus, the other customers connected at the same point are not disturbed. The international standards related to harmonic distortion limits can be classified as follows:

A. Standards specifying limits for individual nonlinear equipment

- IEC 61000-3-2 [7], which specifies the current harmonic limits for low voltage equipment that has an input current less than $16 \mathrm{~A}$.

- IEC 61000-3-12 [8], which specifies the current harmonic limits for an equipment that has an input current between $16 \mathrm{~A}$ and $75 \mathrm{~A}$

- IEC 61800-3 [9],which specifies the electro-magnetic compatibility (EMC) requirements of the adjustable speed drive systems

As noticed, the above standards are for small rating and low voltage harmonic loads only. In addition, the above standards do not set limits on the overall distribution network.

\section{B. Standards specifying limits for electrical networks}

- IEEE 519-1992 [10], this document introduces many useful recommended practices for harmonics control in electrical networks. This document is widely used in the industrial sector and many consultants/clients use the limits indicated in it as contractual limits within their specifications.

- IEC 61000-3-6 [11], this specification performs an assessment of the harmonic emission limits for distorting loads in medium voltage and high voltage power systems. Up till now, this specification is not widely used in the industrial sector because it is rather new (punlished in 2008).

- British engineering recommendation G5/4-1 [12], this document provides some helpful engineering recommendations for establishing the allowable harmonic limits of the voltage distortions in the United Kingdom.

\section{IEEE 519-1992 HARMONIC LIMITS}

\section{A. Harmonic current distortion limits}

Harmonic current distortion limits are introduced in the IEEE 519-1992. A summary of these current harmonic limits is shown in Table I. Setting limits for the current harmonic levels protects the utility company and the other utility consumers connected on the same feeder.

where:

$\mathrm{I}_{\mathrm{SC}}$ : maximum short circuit current at $\mathrm{PCC}$

$\mathrm{I}_{1}$ or $\mathrm{I}_{\mathrm{L}}$ : maximum demand load current (fundamental frequency component) at PCC 


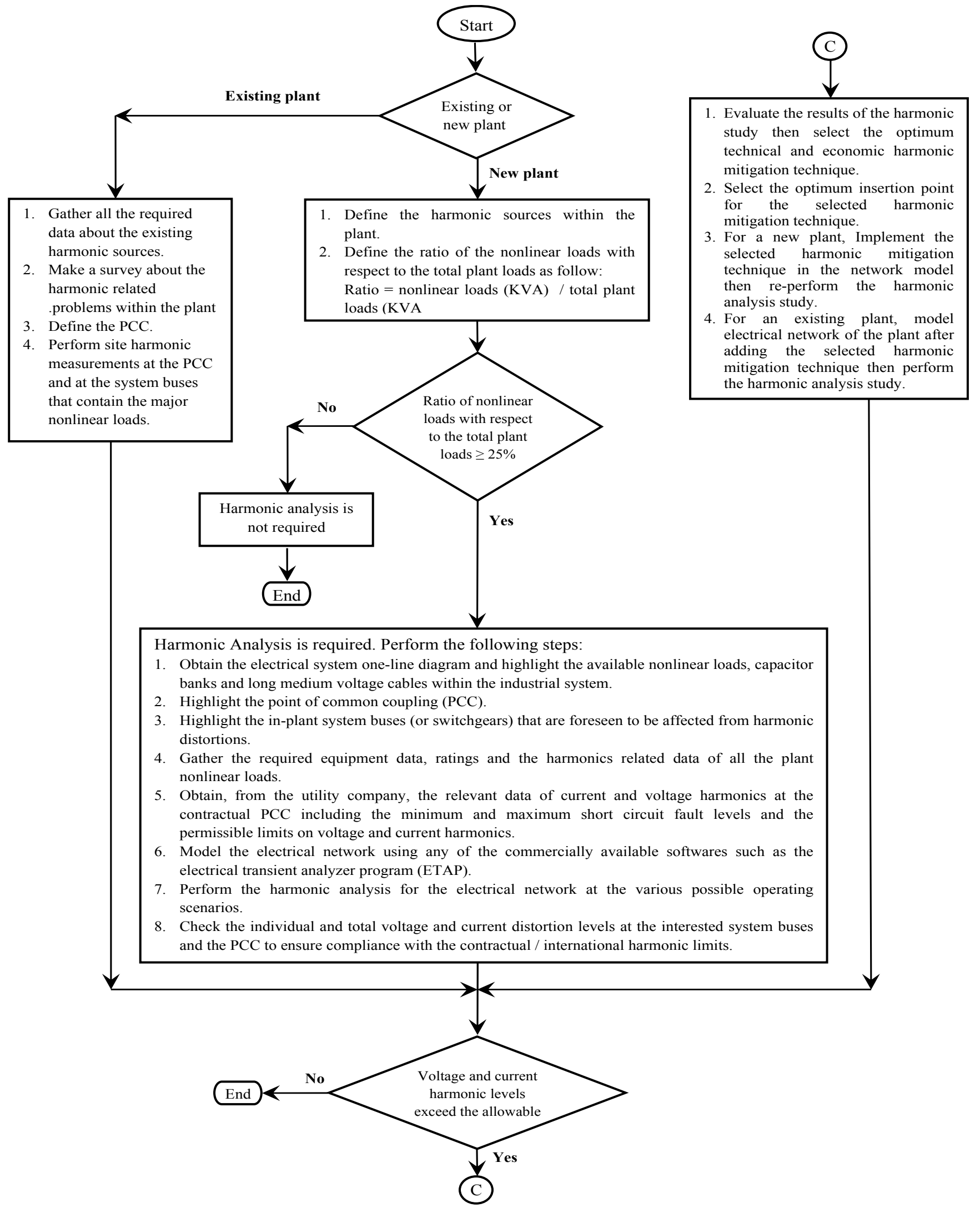

Fig. 4. A comprehensive procedure for the steps required to perform a harmonic analysis 


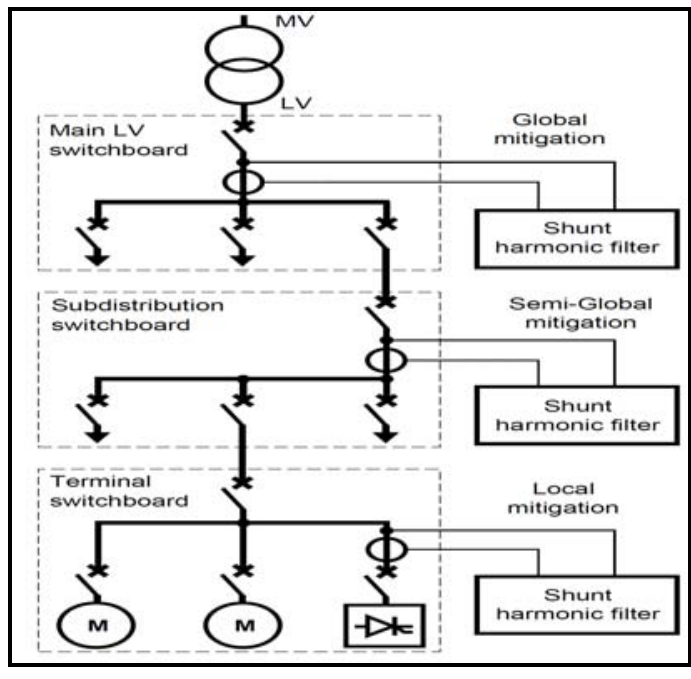

Fig. 5. Various insertion points for the harmonic filters

It should be noted that all the power generation equipment are limited to these values of current distortion, regardless of the actual $\mathrm{I}_{\mathrm{SC}} / \mathrm{I}_{1}$ ratio. The ratio $\mathrm{I}_{\mathrm{SC}} / \mathrm{I}_{\mathrm{L}}$ is the ratio of the short circuit current available at the (PCC) to the maximum fundamental load current. It is recommended that the load current $\left(\mathrm{I}_{\mathrm{L}}\right)$ be calculated over any (15) or (30) min period and then averaged over the next (12) month period.

TABLE I. HARMONIC CURRENT DISTORTION LIMITS FOR GENERAL DISTRIBUTION SYSTEMS (SYSTEM VOLTAGES: FROM 120V TO 69 KV)

\begin{tabular}{|c|c|c|c|c|c|c|}
\hline \multirow{3}{*}{$\mathbf{I}_{\mathrm{SC}} / \mathbf{I}_{1}$} & \multirow{2}{*}{\multicolumn{5}{|c|}{$\begin{array}{c}\text { Odd harmonic order h (\%) } \\
\text { Individual current harmonic distortion (\%) }\end{array}$}} & \multirow{3}{*}{$\begin{array}{c}\text { Total } \\
\text { harmonic } \\
\text { distortion } \\
\text { THD } \%\end{array}$} \\
\hline & & & & & & \\
\hline & $h<11$ & $11 \leq h<17$ & $17 \leq h<23$ & $23 \leq h<35$ & $h \geq 35$ & \\
\hline$<20 *$ & 4 & 2 & 1.5 & 0.6 & 0.3 & 5 \\
\hline $20-50$ & 7 & 3.5 & 2.5 & 1 & 0.5 & 8 \\
\hline $\begin{array}{l}50- \\
100 \\
\end{array}$ & 10 & 4.5 & 4 & 1.5 & 0.7 & 12 \\
\hline $\begin{array}{l}100- \\
1000 \\
\end{array}$ & 12 & 5.5 & 5 & 2 & 1 & 15 \\
\hline$>1000$ & 15 & 7 & 6 & 2.5 & 1.4 & 20 \\
\hline
\end{tabular}

\section{B. Harmonic voltage distortion limits:}

The IEEE 519-1992 defines the allowable voltage harmonic limits at the PCC. Table II summarizes the limits for the low voltage systems and Table III summarizes the limits for the medium and high voltage systems.

Where:

- Special systems: critical applications like hospitals and airports

- Dedicated systems: systems that contain only nonlinear loads

It is important to highlight that the limits listed in Table III should be used as system design values for normal operation conditions (lasting more than one hour). For shorter operation periods, during start-ups or unusual transient conditions, these harmonic limits may be allowed to exceed by $50 \%$.
TABLE II. HARMONIC VOLTAGE DISTORTION LIMITS FOR LOW VOLTAGE DISTRIBUTION SYSTEMS (SYSTEM VOLTAGES: BELOW $1 \mathrm{KV}$ )

\begin{tabular}{|c|c|c|c|}
\hline $\begin{array}{c}\text { Allowable voltage } \\
\text { THD }\end{array}$ & $\begin{array}{c}\text { Special } \\
\text { systems }\end{array}$ & $\begin{array}{c}\text { General } \\
\text { distribution } \\
\text { systems }\end{array}$ & $\begin{array}{c}\text { Dedicated } \\
\text { systems }\end{array}$ \\
\hline Voltage THD (\%) & $3 \%$ & $5 \%$ & $10 \%$ \\
\hline
\end{tabular}

TABLE III. HARMONIC VOLTAGE DISTORTION LIMITS FOR MEDIUM AND HIGH VOLTAGE DISTRIBUTION SYSTEMS

\begin{tabular}{|c|c|c|}
\hline Bus voltage & $\begin{array}{c}\text { Individual voltage } \\
\text { harmonic distortion } \\
(\%)\end{array}$ & $\begin{array}{c}\text { Total voltage } \\
\text { harmonic } \\
\text { distortion (\%) }\end{array}$ \\
\hline $69 \mathrm{kV}$ and below & $3 \%$ & $5 \%$ \\
\hline From $69 \mathrm{kV}$ to $161 \mathrm{kV}$ & $1.5 \%$ & $2.5 \%$ \\
\hline $161 \mathrm{kV}$ and above & $1 \%$ & $1.5 \%$ \\
\hline
\end{tabular}

\section{CONCLUSIONS}

Harmonic analysis studies are necessary to analyze the current and voltage harmonic levels within any industrial electrical system and to check if these levels comply with the contractual or international standard limits. This paper provides a comprehensive approach for performing a harmonic study, presented in the form of a flowchart. In addition, this paper presents the current and voltage harmonic limits used in industrial systems.

\section{REFERENCES}

[1] M. Z. El-Sadek, Power system harmonics, 2nd edition, Mukhtar Press, Egypt, 2007

[2] R. G. Ellis, "Harmonic analysis of industrial power systems", IEEE Transactions on Industry Applications, Vol. 32, No. 2, pp. 417-421, 1996

[3] IEEE Std 399-1997, Recommended practice for industrial and commercial power systems analysis, ANSI/IEEE, 1997

[4] J. P. Nelson, "A Better understanding of harmonics distortions in the petrochemical industry", IEEE Transactions on Industry Applications, Vol. 40, No. 1, pp. 220-231, 2004

[5] S. F. Mekhamer, A. Y. Abdelaziz, S. M. Ismael, "Technical comparison of harmonic mitigation techniques for industrial electrical power systems", MEPCON 2012, Fifteenth International Middle East Power Systems Conference, Paper ID: 214, Alexandria, Egypt, 2012

[6] E. Bettega, J. N. Fiorina, Cahier Technique no. 183: Active harmonic conditioners and unity power factor rectifiers, Schneider Electric, 1st edition, 1999

[7] IEC Std 61000-3-2, Electromagnetic compatibility (EMC)-Part 3-2: Limits for harmonic current emissions (equipment input current $\leq 16 \mathrm{~A}$ per phase, IEC, 2009

[8] IEC Std 61000-3-12, Electromagnetic compatibility (EMC)-Part 3-12: Limits for harmonic currents produced by equipment connected to public low-voltage systems with input current $>16$ a and $\leq 75$ a per phase, IEC, 2011

[9] IEC Std 61800-3, Adjustable speed electrical power drive systems-Part 3: EMC requirements and specific test methods, IEC, 2004

[10] IEEE Std 519, Recommended practice and requirements for harmonics control in electrical power systems, ANSI/ IEEE, 1992

[11] IEC Std 61000-3-6, Electromagnetic compatibility (EMC)-Part 3-6: Assessment of emission limits for the connection of distorting installations to MV, HV and EHV power systems, IEC, 2008

[12] British Engineering Recommendation, Planning levels for harmonic voltage distortion and the connection of nonlinear equipment to transmission systems and distribution networks in the United Kingdom, G5/4-1, 2001 